\title{
Optimal design of triaxial weave fabric composites under tension
}

\author{
Zhenzhou Wang ${ }^{1}$, Jiangbo Bai2 ${ }^{*}$, Adam Sobey ${ }^{1}$, Junjiang Xiong' ${ }^{2}$ Ajit Shenoi ${ }^{3}$
}

1. Fluid Structure Interactions Group, University of Southampton, Southampton, UK

2. School of Transportation Science and Engineering, Beihang University, Beijing, 100191(*, corresponding author: baijiangbo@buaa.edu.cn)

3. Southampton Marine and Maritime Institute, University of Southampton, Southampton, UK

\begin{abstract}
Triaxial weave fabrics are increasingly used in ultralight structures, such as the wings of unmanned aerial vehicles (UAVs) and deployable antenna on spacecraft. The tensile strength to stiffness ratio for these applications is important, requiring an optimal weave pattern; in this paper Genetic Algorithms are used to improve these designs. The mechanical response is obtained using the minimum total complementary potential energy principle where the yarns are approximated as curved beams in a micromechanical unit cell. Leading Genetic Algorithms are benchmarked to determine which perform best. The results form a disconnected Pareto front where the left hand part can be used for flexible structures but is difficult to find. An overall improvement in strength to stiffness ratio of $1191 \%$ is made with 643 designs found better than a current example. The selection of the Genetic Algorithm is shown to be crucial with only MLSGA-NSGAII regularly finding the entire Pareto front.
\end{abstract}

Keywords: Triaxial weave fabric (TWF); Multi-objective optimisation; Tensile mechanical properties; Genetic Algorithms 


\section{Introduction}

Novel ultralight applications are creating a demand for new materials. These new materials need to have good mechanical properties despite the low mass requirement. Triaxial weave fabrics (TWF), illustrated in Figure 1, are an example of materials finding growing usage in these structures. They are composites with longitudinal fibres in three directions, $0^{\circ}$ and $\pm 60^{\circ}$, which provide mechanically quasi-isotropic properties, are lightweight due to the high degree of porosity and reduce the impact from air loads. It is also possible to design these structures with a small number of layers, as low as 1 . The tensile strength to stiffness ratio is the most important mechanical property in many applications of triaxial weave fabric composites, especially for deployable antenna on spacecraft and ultra-thin wing skins of unmanned aerial vehicles (UAVs) as these properties provide flexible structures that are damage resistant. The crimp, or undulation, of the yarns significantly influences the mechanical properties and requires an optimal weave pattern to maximise the strength to stiffness ratio. However, it is not fully known how close the currently available fibre design schemes are to optimal, since these materials are relatively new.

Genetic Algorithms (GAs) are popular tools for finding optimal composite designs. A review of Composite Structures, Composites Part A, Composites Part B and Composites Science and Technology shows 214 papers utilising Genetic Algorithms to optimise composite structures and materials since 2008. The optimisation problems can be classified into single objective, weighted multi-objective, reducing a multiple objectives problem down to one objective, and multi-objective problems. Multi-objective problems represent the most interesting set as they provide an engineer with a greater understanding of the design space; 39 of the papers found focus on these problems by generating Pareto fronts. Single objective or weighted average problems tend to be easier to solve so a wider range of Genetic Algorithms are capable of solving the problems especially if combined with variable spaces that are small and/or simple.

It is essential to utilise a suitable algorithm for solving an optimisation problem. The "no free lunch' theorem states that an algorithm that improves its performance on a category of problems inevitably degrades its performance on other types; optimisation algorithms are designed to be specialist to a problem type or have lower performance across all problems. Therefore, a variety of Genetic Algorithms have been developed to solve multi-objective problems categorised by their performance on different types of problems. As an example to demonstrate the importance of selecting the correct algorithm, Mutlu et al. [1] benchmark the 
performance of a number of popular algorithms on a composite grillage optimisation problem. The problem has limited input variables but even this simple problem demonstrates the need for state-of-the-art algorithms to evolve the entire Pareto front, and that these should be specialist algorithms reflecting the problem type. Reviewing the multi-objective optimisation papers, where a Pareto front is developed, the most popular Genetic Algorithm was NSGA-II but a number of older algorithms are still prevalent in this literature. However, in much of the literature the names of the Genetic Algorithms used are not stated, making it difficult to assess the validity of the results.

In addition to the algorithm selection, the hyper-parameters, such as population size, number of generations and mutation and crossover type, affect the performance. From the reviewed literature most of the composite material/structural multi-objective optimisation cases utilise population sizes approximating 600 individuals; this is consistent with the computer science literature where the popular algorithms selected for comparison in the CEC'09 benchmarking use this value or smaller [2]. The reviewed literature generally uses 100 generations or less, totalling 60,000 function calls, including the reviewed woven roving optimisation literature [36], with some papers using as few as 350 function evaluations [7]. There is a tendency for the number of function calls to be poorly documented in the composite material/structural optimisation literature and it is suspected that many use smaller numbers to reduce computational time but which may compromise the quality of the final solution. Additionally, the number of repeated independent run cycles is not stated in many papers, with a focus on fewer long runs, indicating that the optimisation results were obtained from one run making it difficult to determine the consistency of the results.

The literature shows promising properties for Triaxial Weave Fabric composites but there is no consensus on which weave patterns provide optimal mechanical characteristics, for example high strength to stiffness ratios. The literature indicates that genetic algorithms are a popular method for optimising composite materials and structures but non-specialist Genetic Algorithms are utilised, many of which are out of date, on single objective or weighted multiobjective problems. It is proposed that current composite structural problems are becoming too complex for these non-specialist algorithms, leading to unresolved Pareto fronts. However, the selection of the correct Genetic Algorithm is difficult as the evolutionary computation literature is not categorised in a manner that reflects composite structures, defining the dominant categories as only constrained or unconstrained formulations for static multi-objective 
optimisation. Therefore the current study benchmarks state-of-the-art Genetic Algorithms on a multi-objective problem to find optimal designs, Pareto fronts, for TWF composites. The Genetic algorithms considered for the benchmarking are a specialist constrained, MLSGANSGAII, a specialist unconstrained, MOEA/D, the most popular, NSGA-II, and one population based local search method, MTS, which demonstrates generally good performance over both formulation types.

\section{TWF model for tensile strength and modulus}

The tensile modulus and strength of the TWF composites are predicted using the minimum total complementary potential energy principle developed by Bai et al. [8]. Figure 1 shows the geometry parameters of a unit cell of a TWF composite with the idealized undulation shape of the yarn shown in comparison to a micrograph of the actual undulation. The undulating neutral axis of the triaxial yarns is expressed using a sinusoidal function. The tensile loading is along the 0 degree yarn direction, where the internal forces and bending moments are shown in Figure 2.

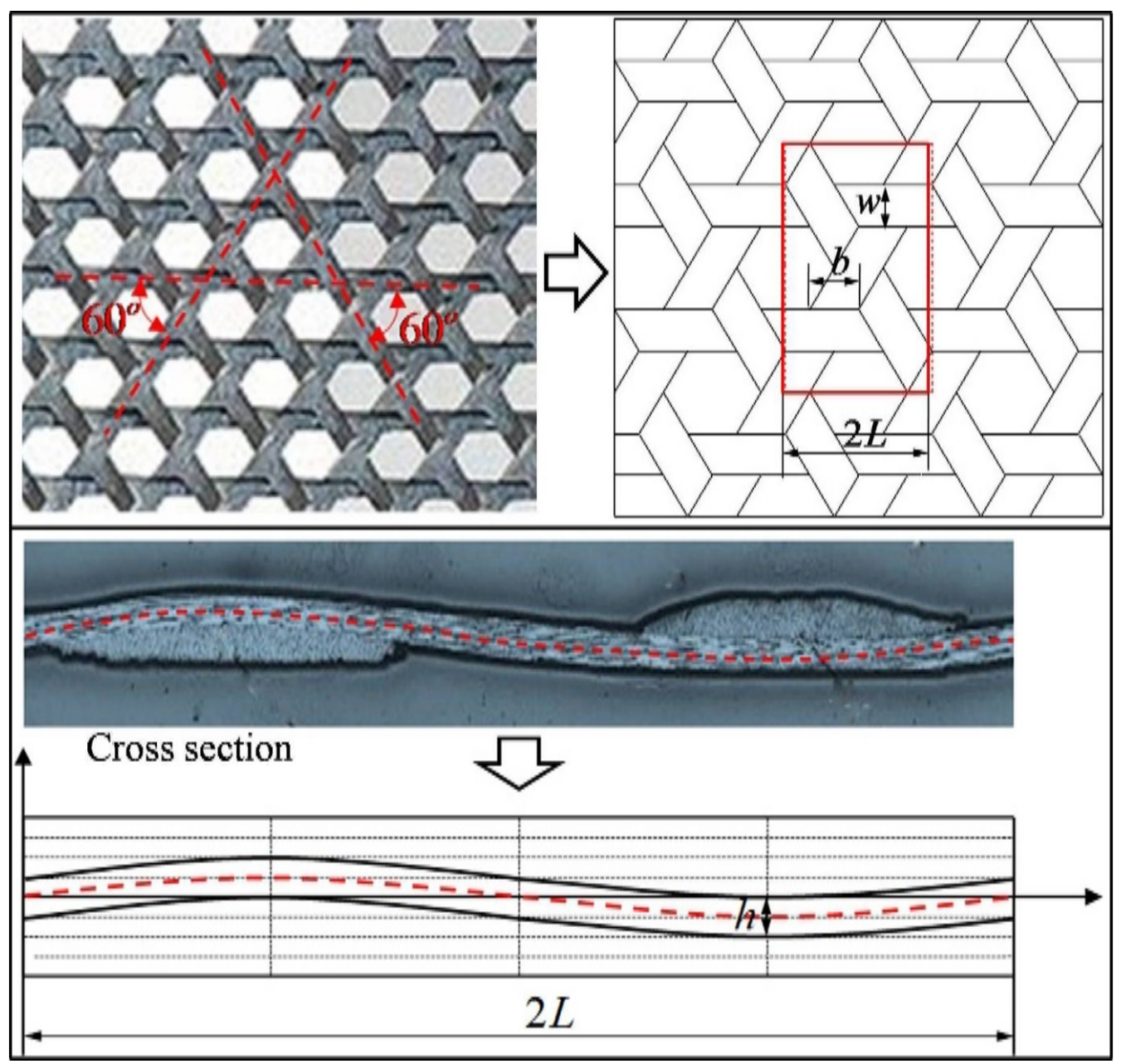


Fig. 1 Unit cell and micrograph of TWF composites [8]

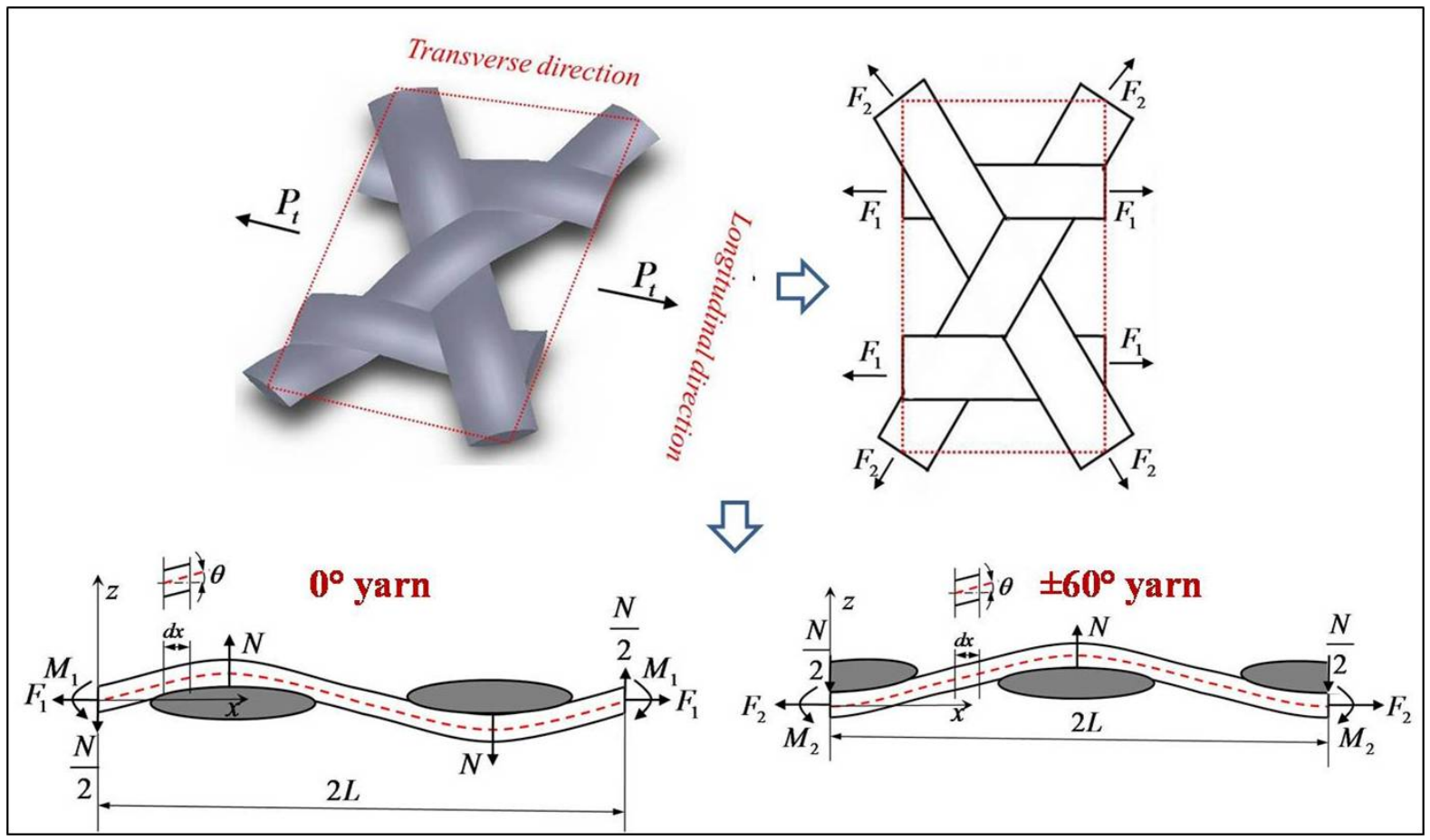

Fig. 2 Internal forces and bending moments on a unit cell [8]

We refer to Bai et al. [8] for a detailed introduction to the analytical model. In brief the tensile modulus, $E_{T}$, is expressed in equation 1 as,

$E_{T}$

$$
\begin{aligned}
& =\frac{P_{T}}{2 \sqrt{3} \delta_{T} h}=\frac{1}{2 \sqrt{3} h}\left[4\left(B_{1}+B_{4}\right) C_{1}^{2}+8\left(B_{5}+B_{11}\right)\left(0.5-C_{1}\right)^{2}+4\left(B_{2}+2 B_{6}\right) C_{2}^{2}+8 B_{7} C_{3}^{2}+4 B_{3}\right. \\
& \left.C_{1} C_{2}+8 B_{8} C_{2}\left(0.5-C_{1}\right)+8 B_{9} C_{3}\left(0.5-C_{1}\right)+8 B_{10} C_{2} C_{3}\right]^{-1}
\end{aligned}
$$

and strength per unit length, $X_{t}$, in equation 2 as,

$$
X_{t}=\frac{\min \left(P_{t f 1}, P_{t f 2}\right)}{4 \sqrt{3} h L},
$$

where the $P_{t f 1}$ and $P_{t f 2}$ are the internal tensile loading along the 0 degree and \pm 60 degrees yarns. $B_{i}(i=1,2, \cdots, 11)$ and $C_{i}(i=1,2,3)$ are the transformation variables defined in Bai et al. $[8]$.

In order to validate the analytical model the predictions are compared to mechanical properties 
taken from experiments performed by Kueh and Pellegrino [9] for T300/Hexel8552, Aoki and Yoshida [10] and Aoki et al. [11] for T300/NM35 and Zhao et al. [12] for a carbon fibre/epoxy resin; the fabric specifications are shown in Table 1. The comparison between the experimental results of the three types of TWF composites are compared to the analytical method in Table 2. The maximum error for the prediction of the tensile modulus and strength compared to the experiments are respectively $13.77 \%$ and $3.40 \%$. The model provides an adequate prediction for the tensile modulus and strength analysis of TWF composites.

Table 1 Manufacturer's data for fabric schema and yarn mechanical properties

\begin{tabular}{|c|c|c|c|c|c|c|}
\hline & $\begin{array}{c}w / \\
\mathrm{mm}\end{array}$ & $\begin{array}{c}h / \\
\mathrm{mm}\end{array}$ & $\begin{array}{c}L / \\
\mathrm{mm}\end{array}$ & $\begin{array}{c}E / \\
\mathrm{MPa}\end{array}$ & $\begin{array}{c}X_{t 0} / \\
\mathrm{MPa}\end{array}$ \\
\hline T300/Hexel8552[9] & 0.803 & 0.078 & 1.56 & 153085 & 4408 & 2296 \\
\hline $\mathrm{T}^{\left[900 / N M 35^{[10,11]}\right.}$ & 0.89 & 0.07 & 1.55 & 176000 & 6860 & 2673 \\
\hline Carbon fibre/epoxy resin $^{[12]}$ & 0.85 & 0.07 & 1.59 & 338570 & 5610 & 3400 \\
\hline
\end{tabular}

Table 2 Verification of tensile modulus and strength predictions from analytical model

\begin{tabular}{|c|c|c|c|c|}
\hline & & T300/Hexel8552[9] & T300/NM35 $5^{[10,11]}$ & Carbon fibre/epoxy resin ${ }^{[12]}$ \\
\hline \multirow{3}{*}{$\begin{array}{l}\text { Modulus } \\
E_{\mathrm{T}}(\mathrm{GPa})\end{array}$} & Experiments & 13.53 & 22.01 & 32.24 \\
\hline & Predictions & 14.93 & 18.97 & 35.23 \\
\hline & Error & $10.29 \%$ & $13.77 \%$ & $9.27 \%$ \\
\hline \multirow{3}{*}{$\begin{array}{l}\text { Strength } \\
X_{t}(\mathrm{MPa})\end{array}$} & Experiments & 165.71 & 218.43 & 239.43 \\
\hline & Predictions & 161.86 & 210.36 & 247.29 \\
\hline & Error & $2.32 \%$ & $3.40 \%$ & $3.28 \%$ \\
\hline
\end{tabular}

\section{Multi-objective design methodology}

The shape of the objective space and its relationship with the variable space is currently unknown for TWF composites under tensile loading. This means that the problem type is not defined and that the corresponding algorithms suitable to that problem type cannot be selected. In this case the CEC'09 benchmarking [2] is used to select the algorithms for comparison: NSGA-II which has generally good performance across all problem sets, MOEA/D for unconstrained problems and MTS which came second and third on unconstrained and 
constrained problems; in addition a more recently developed Genetic Algorithm, MLSGANSGAII, is also included which performs well on constrained problems. Better constrained performance is shown by DMOEA-DD and LiuLi algorithms but the available versions of the code cannot be converted to solve new problems and the documentation of the original algorithms is not enough to reproduce them.

A general schematic diagram of the optimisation process is illustrated in Figure 3. The four Genetic Algorithms are substituted into the selection and reproduction stage, where MTS has no crossover and mutation mechanisms. All individuals from the population are evaluated through the analytical model. In order to perform a fair test across the different Genetic Algorithms the same genetic operator types: selection, crossover and mutation, use the same operator rate, which is the same as those selected for the CEC'09 benchmarking [13]. MTS is a population based optimisation algorithm with a different set of hyper-parameters; these are kept the same as in the CEC'09 competition, except the population size and total number of function calls which are kept the same as the other three algorithms.

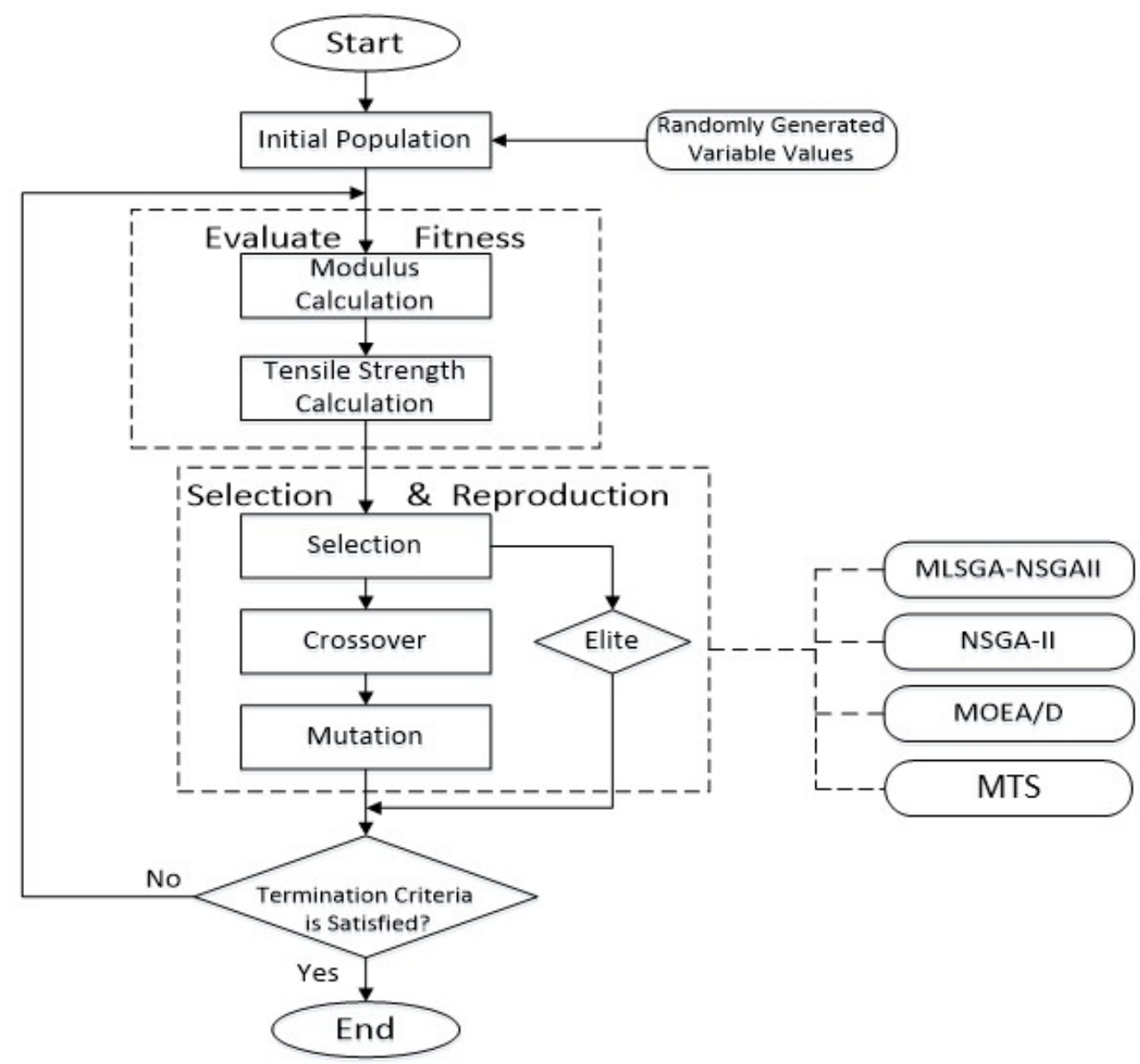

Fig. 3 General optimisation procedure 


\subsection{Formulation of multi-objective optimisation problem}

The multi-objective optimisation problem is formulated in both a constrained and unconstrained format for the TWF composite material in Equations 3 and 4,
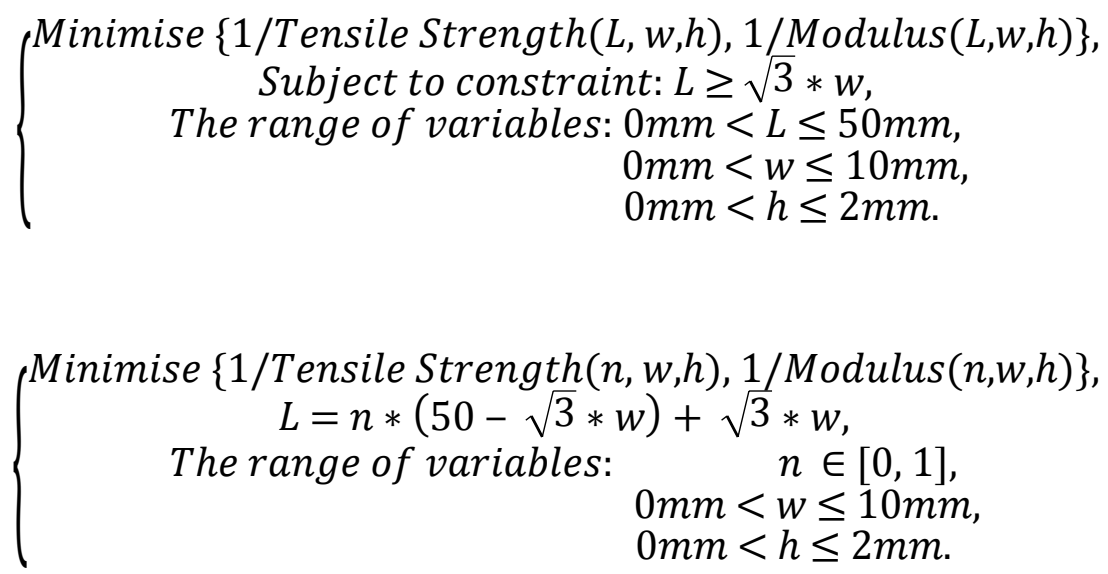

These are to provide a material with a maximum strength and modulus under tensile load. T300/Hexel8552 is selected as the combination to be optimised as the most mature TWF composite. The variables yarn undulation length, L, yarn width, w, and height, $\mathrm{h}$, are the parameters influencing the strength and modulus in the analytical model which are shown in Figure 1. The ranges of these variables are selected to ensure suitability for a range of existing applications for TWF composites. A constraint is in place to ensure the weave patterns can be manufactured. Current manufacturing precision can be controlled to $10^{-2}$ millimetres; this can be extended to three decimal places but is more expensive. The interval between variables has been selected at $10^{-10}$ millimetres, substantially beyond the capability of current manufacturing, because the optimisation procedure seeks to fully document the objective space and uses an extended search space to benchmark the different optimisation algorithms.

\subsection{NSGA-II}

NSGA-II is the most commonly used Genetic Algorithm due to its success as a general solver with good performance across a range of problem types. It is commonly applied in the optimisation of composite materials and structures. The algorithm was first published by Deb et al. [14] in 2002 and is based on non-domination of solutions; if a solution is not simultaneously dominated on all objectives by any other solutions in the population, the solution is defined as non-dominated. Solutions not dominated by each other are classified into 
the same non-domination rank. The previous non-domination ranks are temporarily discounted when sorting out a new rank. We refer to Deb et al. [14] for a detailed introduction to NSGAII. The NSGA-II parameters used within this optimisation are listed in Table 3 where the selection, crossover and mutation types are default settings.

Table 3 NSGA-II parameter definition

\begin{tabular}{|c|c|c|}
\hline \multirow{2}{*}{ Initialisation } & Initialisation type & Random \\
\cline { 2 - 3 } & Encoding type & Real value coded \\
\hline \multirow{4}{*}{ Reproduction } & Selection type & NSGAII - crowding distance \\
\cline { 2 - 3 } & Crossover type & Real variable SBX \\
\cline { 2 - 3 } & Crossover rate & 0.7 \\
\cline { 2 - 3 } & Mutation type & Polynomial mutation \\
\cline { 2 - 3 } & Mutation rate & 0.08 \\
\hline \multirow{2}{*}{ Termination } & Stopping criteria & Total number of iterations are \\
& & \multicolumn{2}{c}{ reached } \\
\hline
\end{tabular}

\subsection{MOEA/D}

MOEA/D is the top performer on unconstrained problems and was first published in Zhang and $\mathrm{Li}$ [15] in 2007. This algorithm is based on weight vector and decomposition, splitting the problem into a number of sub-problems and optimising each sub-problem separately. This algorithm is complex for the user as substantially different Pareto optimal solutions can be obtained by changing the weight vector. Since each generation uses only 5 to 10 percent of the total population size in MOEA/D, the generation number is much higher when compared to NSGA-II and MLSGA-NSGAII with the same population size and total function calls. For detailed information about MOEA/D, we refer to Zhang and Li [15]. The MOEA/D parameters are summarised in Table 4. The selection, crossover and mutation types are MOEA/D default settings with default settings for crossover rate but the mutation rate is kept the same as NSGAII and MLSGA-NSGAII.

Table 4 MOEA/D parameter definition

\begin{tabular}{|c|c|c|}
\hline \multirow{2}{*}{ Initialisation } & Initialisation type & Random \\
\cline { 2 - 3 } & Encoding type & Real value coded \\
\hline Reproduction & Selection type & MOEA/D \\
\hline
\end{tabular}




\begin{tabular}{|c|c|c|}
\hline \multirow{2}{*}{} & Crossover type & Multiple parents \\
\cline { 2 - 3 } & Crossover rate & 1 \\
\cline { 2 - 3 } & Mutation type & Polynomial mutation \\
\cline { 2 - 3 } & Mutation rate & 0.08 \\
\hline \multirow{2}{*}{ Termination } & Stopping criteria & $\begin{array}{c}\text { Total number of iterations are } \\
\text { reached }\end{array}$ \\
\hline
\end{tabular}

\subsection{MLSGA-NSGAII}

MLSGA was first introduced by Grudniewski and Sobey [16] in 2017 and introduces a collective level reproduction mechanism, in addition to the individual level used in standard genetic algorithms, and a split fitness function. MLSGA-NSGAII is a hybrid Genetic Algorithm that utilises the crowding distance of NSGA-II as the individual reproduction step in MLSGA. It is shown to have improved performance over NSGA-II on constrained and unconstrained problems, showing particularly strong performance on constrained problems. We refer to Grudniewski and Sobey [16] for information about MLSGA in details. Table 5 summarises the specific parameters of MLSGA-NSGAII in the current paper. The selection, crossover and mutation types and rates are MLSGA-NSGAII default settings.

Table 5 MLSGA-NSGAII parameter definition

\begin{tabular}{|c|c|c|}
\hline \multirow{2}{*}{ Initialisation } & Initialisation type & Random \\
\hline & Encoding type & Real value coded \\
\hline \multirow{2}{*}{ Classification } & Classification method & Support Vector Machine \\
\hline & Number of collectives & 8 \\
\hline \multirow{7}{*}{ Individual Level } & Individual fitness evaluation & MLSU \\
\hline & Individuals selection type & NSGAII - crowding distance \\
\hline & Individuals crossover type & Real variable SBX \\
\hline & Crossover rate & 0.7 \\
\hline & Individuals mutation type & Polynomial mutation \\
\hline & Mutation rate & 0.08 \\
\hline & Elite rate & 0.1 \\
\hline \multirow[b]{2}{*}{ Collective Level } & Collective fitness evaluation & MLSU \\
\hline & $\begin{array}{c}\text { Number of collectives } \\
\text { elimination }\end{array}$ & 1 \\
\hline
\end{tabular}




\begin{tabular}{|c|c|c|}
\hline & Number of new collectives & 1 \\
\cline { 2 - 3 } & $\begin{array}{c}\text { Total number of skipped } \\
\text { collectives elimination }\end{array}$ & $25 \%$ of generation number \\
\hline Termination & Stopping criteria & $\begin{array}{c}\text { Total number of iterations are } \\
\text { reached }\end{array}$ \\
\hline
\end{tabular}

\subsection{MTS}

MTS was first introduced by Tseng and Chen [17] in 2007 and shows good performance on both unconstrained and constrained multi-objective optimisation problems. This algorithm is a population based local search method. The initial solutions are randomly generated where the local search starts around each solution. We refer to Tseng and Chen [17] for detailed information about MTS. The MTS parameters are listed in Table 6 which are set to the default values from the literature. The same number of function calls with the same size of initial population as the other algorithms are used.

Table 6 MTS parameter definition

\begin{tabular}{|c|c|c|}
\hline \multirow{2}{*}{ Initialisation } & Initialisation type & Random \\
\cline { 2 - 3 } & Number of initial solutions & Population size \\
\hline \multirow{4}{*}{ Reproduction } & Local search grade bonus 1 & 9 \\
\cline { 2 - 3 } & Local search grade bonus 2 & 2 \\
\cline { 2 - 3 } & Number of local search test & 5 \\
\cline { 2 - 3 } & Number of local search & 45 \\
\cline { 2 - 3 } & Number of foreground & $\begin{array}{c}\text { 0.125 Population size } \\
\text { Termination }\end{array}$ \\
& Stopping criteria & $\begin{array}{c}\text { Total fitness evaluation } \\
\text { number reached }\end{array}$ \\
\hline
\end{tabular}

\section{Optimisation of TWF composites}

\subsection{Benchmarking of Genetic Algorithms}

A study of population size is used to benchmark the different algorithms on the TWF problem. Many examples in the composite structures literature use generation sizes of 100 or less; which is selected for this investigation. In order to determine the optimal population size for each algorithm, population sizes of 200,600,1000, 1500, 2000 and 3000 were compared for the four selected algorithms using both unconstrained and constrained formulations. All four 
solvers achieve better results on the unconstrained formulation and so only these are documented. The runs generating the best Pareto front from the 30 simulations are illustrated in Figure 4 for the highest and lowest population sizes for each of the four algorithms.

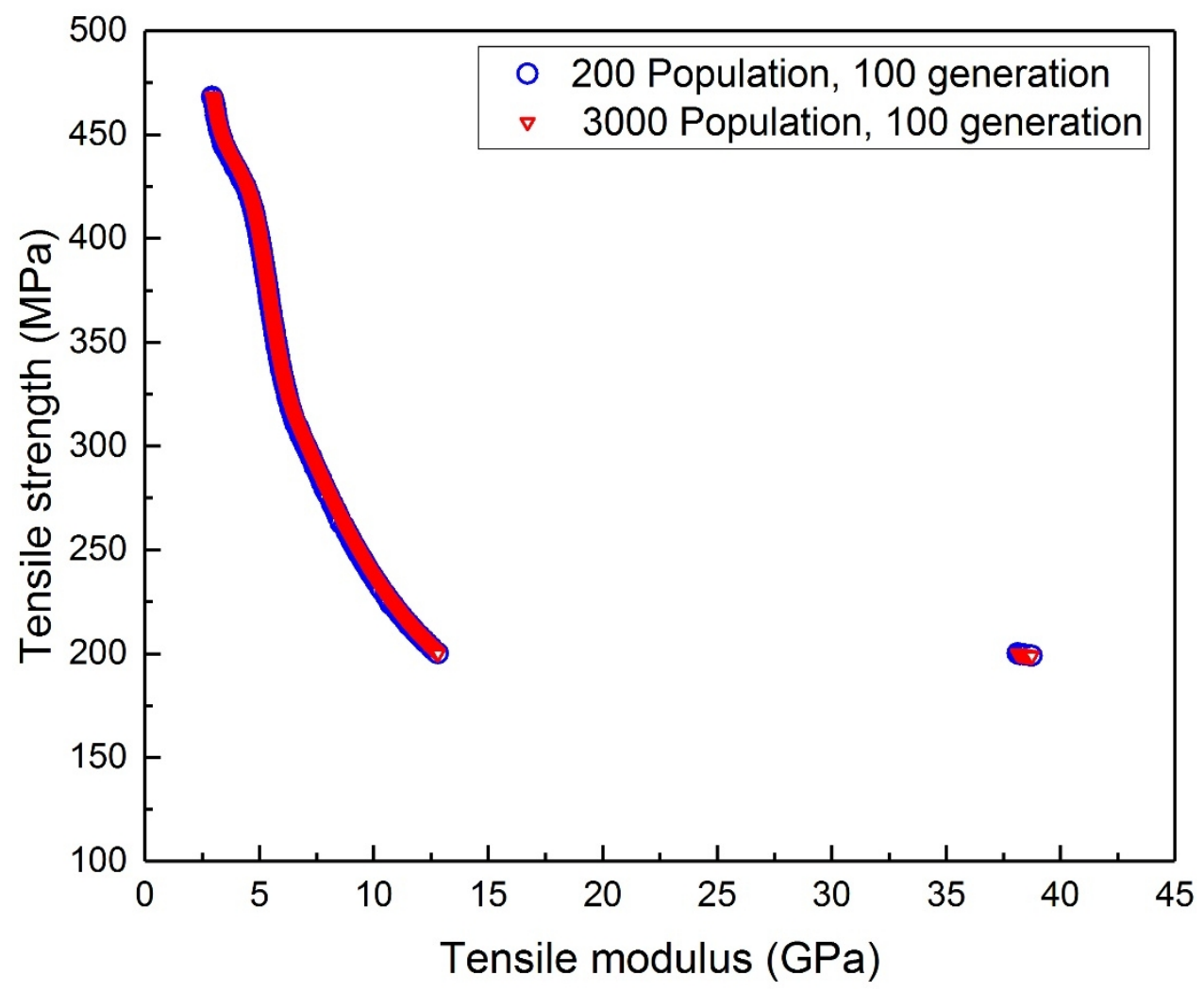

(a)




(b)

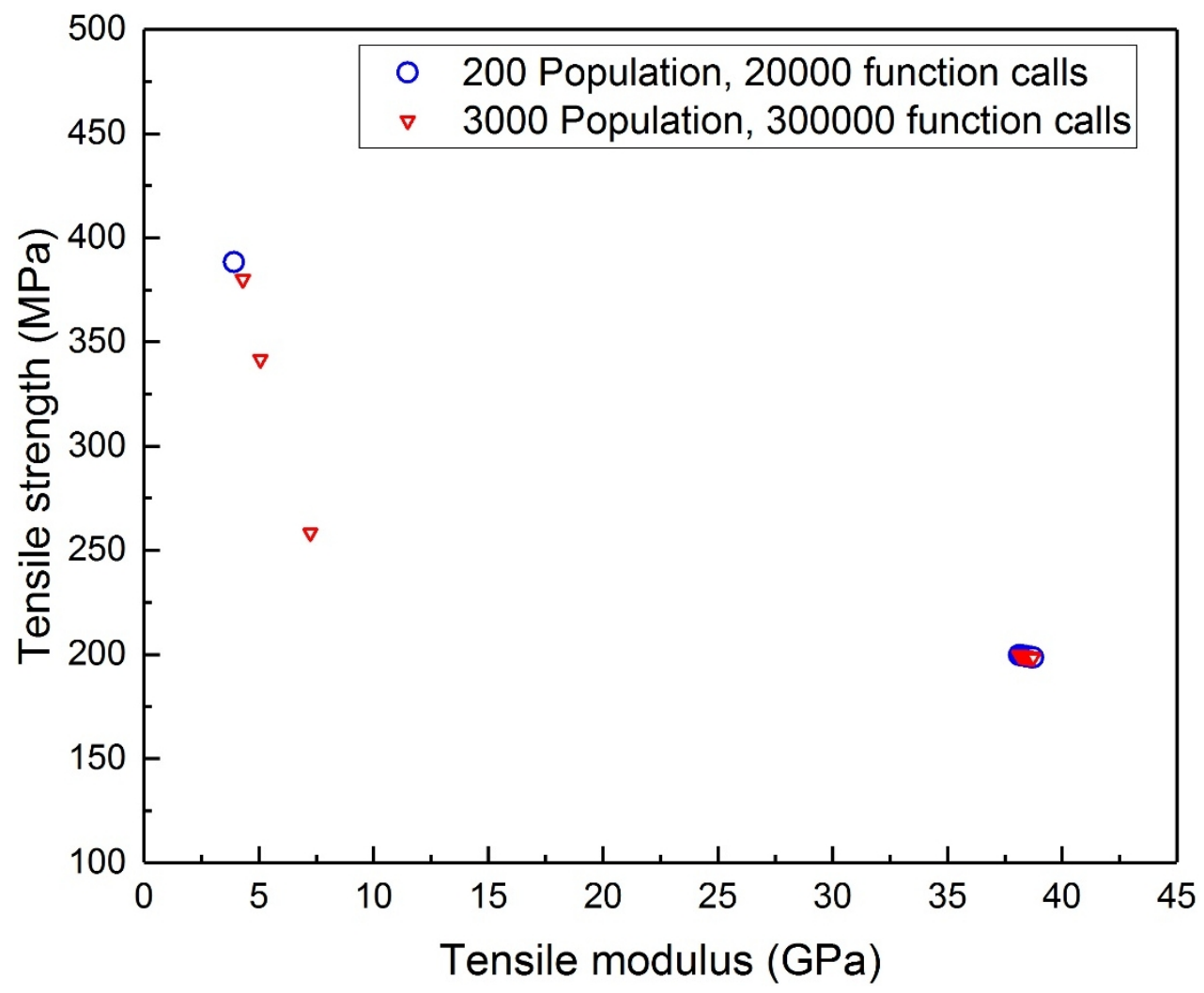

(c)

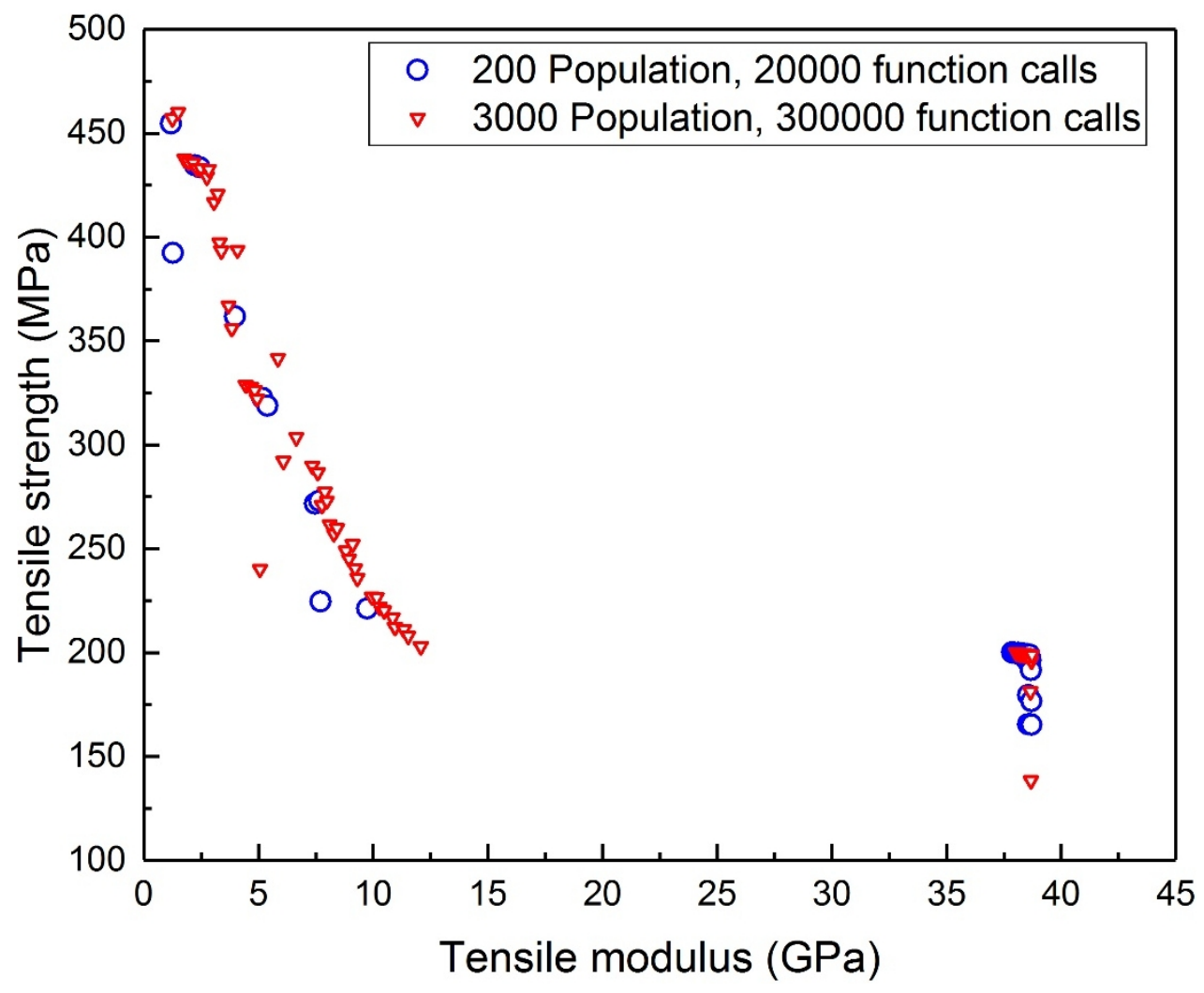

(d)

Fig. 4 Comparison of Pareto fronts for different populations sizes: (a) NSGA-II (b) MLSGA- 
MLSGA-NSGAII and NSGA-II both find the disconnected Pareto front with a good spread of results, covering a similar range, but with little difference in terms of the population size. MTS is expected to obtain good results on both problem formulations but it performs poorly, finding few points on the left side of the Pareto front. MOEA/D performs the worst among the four algorithms with only a small number of points near the Pareto front, despite being a specialist algorithm for unconstrained problems. The population size has a large influence on the quality of the best optimisation results from MTS, unlike the other algorithms. Since MTS and MOEA/D show such poor performance, they are not discussed further.

\subsection{Usability of the Pareto front}

The results in Figure 4 show the best obtained results which show a disconnected Pareto front with two main fronts, which are not equally easy to capture. The front on the right side, with higher tensile moduli, is easier to capture but exhibits lower strengths under tension. The left side has higher strength and lower modulus weave patterns but none of the algorithms can always obtain these results. Therefore the robustness of the algorithms is investigated here determining how regularly they capture the entire Pareto front.

The influence of population size on the frequency of finding the entire disconnected Pareto front is studied, with 600 individuals selected as a representative value from the reviewed literature and is compared against a larger value of 1500. The frequencies for finding the two separated Pareto fronts from 30 runs and the maximum points found on the left side of the Pareto front for each algorithm are summarised in Table 7, where the Pareto fronts are extracted every 50 generations from each run until 200 generations.

Table 7 Success finding the entire Pareto front over 30 cycles

\begin{tabular}{|c|c|c|c|c|c|}
\hline \multicolumn{2}{|c|}{} & \multicolumn{2}{c|}{$\begin{array}{c}\text { Max. points found on left side PF } \\
\text { (total PF storage of 1000) }\end{array}$} & \multicolumn{2}{c|}{ Frequency } \\
\hline $\begin{array}{c}\text { Population } \\
\text { Size }\end{array}$ & Generation & NSGA-II & MLSGA-NSGAII & NSGA-II & MLSGA-NSGAII \\
\hline 1500 & 50 & 808 & 508 & 17 & 26 \\
\hline 1500 & 100 & 828 & 568 & 17 & 26 \\
\hline 1500 & 150 & 873 & 637 & 17 & 27 \\
\hline
\end{tabular}




\begin{tabular}{|c|c|c|c|c|c|}
\hline 1500 & 200 & 964 & 643 & 17 & 28 \\
\hline 600 & 50 & 806 & 393 & 6 & 11 \\
\hline 600 & 100 & 908 & 418 & 6 & 14 \\
\hline 600 & 150 & 960 & 418 & 6 & 15 \\
\hline 600 & 200 & 969 & 547 & 6 & 16 \\
\hline
\end{tabular}

Both algorithms achieve 1000 points across the entire Pareto front for every run. In all cases MLSGA-NSGAII finds the disconnected Pareto front more frequently than NSGA-II. MLSGA-NSGAII shows a greater probability of finding the entire front after a greater number of generations whereas the frequency of finding the disconnected Pareto front through NSGAII does not change after 50 generations. It is found that the ability of the algorithm to find the whole Pareto front is higher with larger population sizes, even for the same number of total function evaluations. This shows that the size of the population, which is proposed here to be larger than the values normally used, is important in achieving the best results for both NSGAII and MLSGA-NSGAII.

In the cases where NSGA-II finds the left hand front it obtains more points than MLSGANSGAII. The number of points on the left side Pareto front increases with higher generations in both NSGA-II and MLSGA-NSGAII. To determine the quality of the Pareto front on the runs that successfully find the left side front, a convergence study is performed on NSGA-II and MLSGA-NSGAII at a population size of 1500. Since the real Pareto front is unknown for these cases a mimicked inverted generational distance (mIGD) is derived by generating the best Pareto front from all of the available data. The mimicked IGD value is based on IGD [2], which quantifies the quality and diversity of a Pareto front by representing the average value of distances from each point on the real Pareto front to its closest point on the obtained Pareto front. In this case a mimicked Pareto front is achieved by filtering all of the dominated and duplicated Pareto front solutions from both MLSGA-NSGAII and NSGA-II. mIGD is defined in equation 5 ,

$$
\operatorname{migD}\left(O, M^{*}\right)=\frac{\sum_{v \in M} d(v, O)}{\left|M^{*}\right|}
$$

where $M^{*}$ is a set of points along the mimicked Pareto front, $\mathrm{O}$ is a set of points on the currently obtained Pareto front, $\mathrm{v}$ represents each point in the set $M^{*}$ and $d(v, O)$ is the minimum Euclidean distance between $\mathrm{v}$ and the points in O; lower mIGD values reflect a better quality 
and diversity of the obtained Pareto front. The mIGD values are calculated only for the cases which achieve the entire Pareto front, and are illustrated in Figure 5. The results show that MLSGA-NSGAII converges five times faster than NSGA-II, which converges at 250 generations. All of the mean mIGD values from MLSGA-NSGAII are lower than that of NSGA-II, indicating a better accuracy and diversity of the front. The best cases from MLSGANSGAII are better than the best from NSGA-II and the worst cases also achieve lower mIGD values than the worst NSGA-II cases until 200 generations. MLSGA-NSGAII shows a considerable improvement in the probability of finding the left side front, with a slightly smaller density of points, but with better quality, diversity and faster convergence.

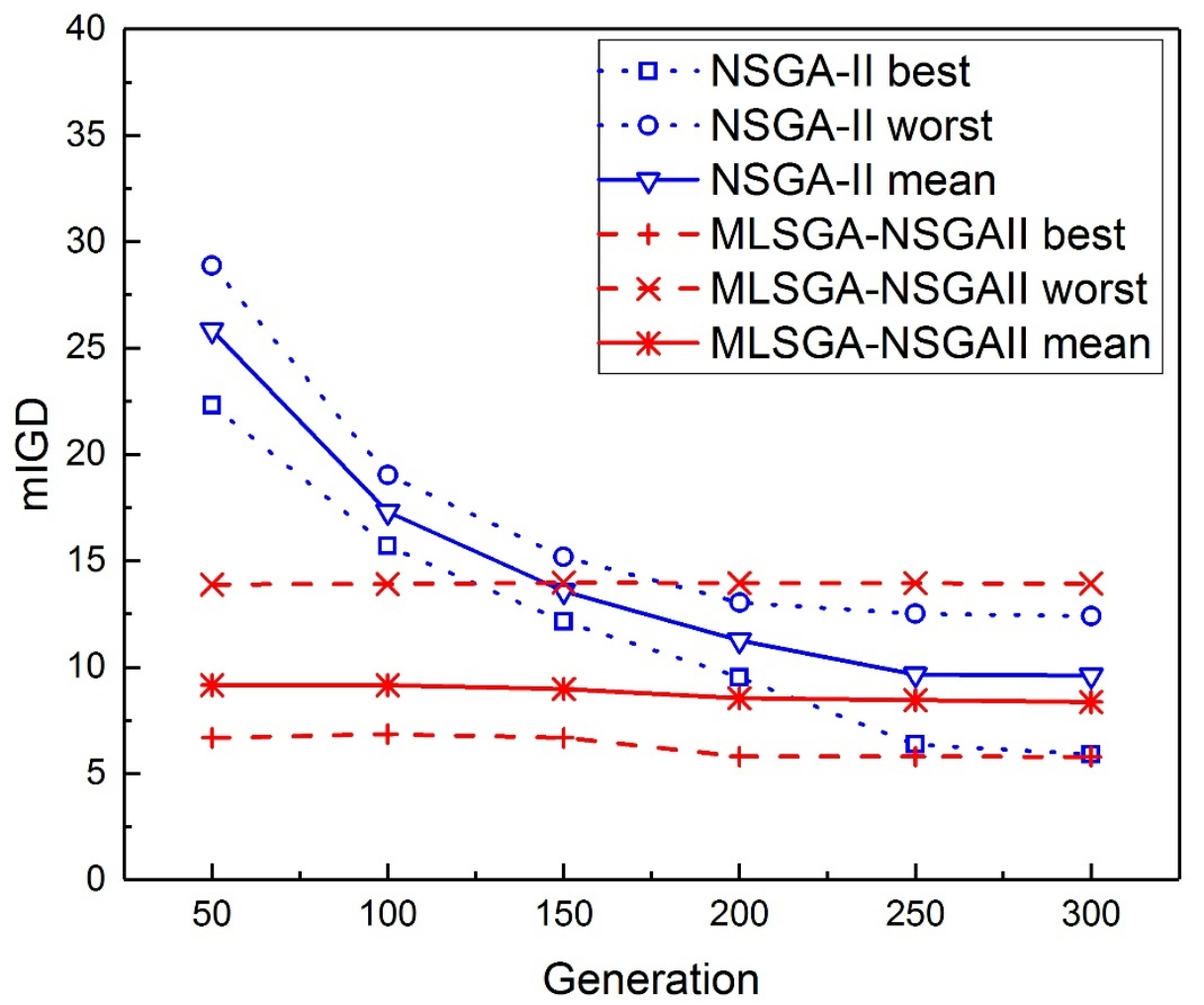

Fig. 5 Comparison of the mIGD values for NSGA-II and MLSGA-NSGAII

\subsection{Design schema for TWF composites}

The results for the MLSGA-NSGAII algorithm with 1500 population size and 200 generations are chosen to demonstrate the implications for TWF composite design, as they have the best performance. Due to the repeatability of the results across the algorithms and number of simulations, each point on the Pareto front is assumed close to the optimal design for the TWF composites. Three points on the disconnected Pareto front were randomly selected by hand to demonstrate the three different types of designs; their location in the objective space and the 
resulting topologies are shown in Figure 6. Further examples of how changes in objective and variable space vary are summarised in Appendix A which shows that the relationship between the objectives and variables is nonlinear on the left hand front, making design decisions more difficult but offering a wide range of potential designs, but linear on the right.

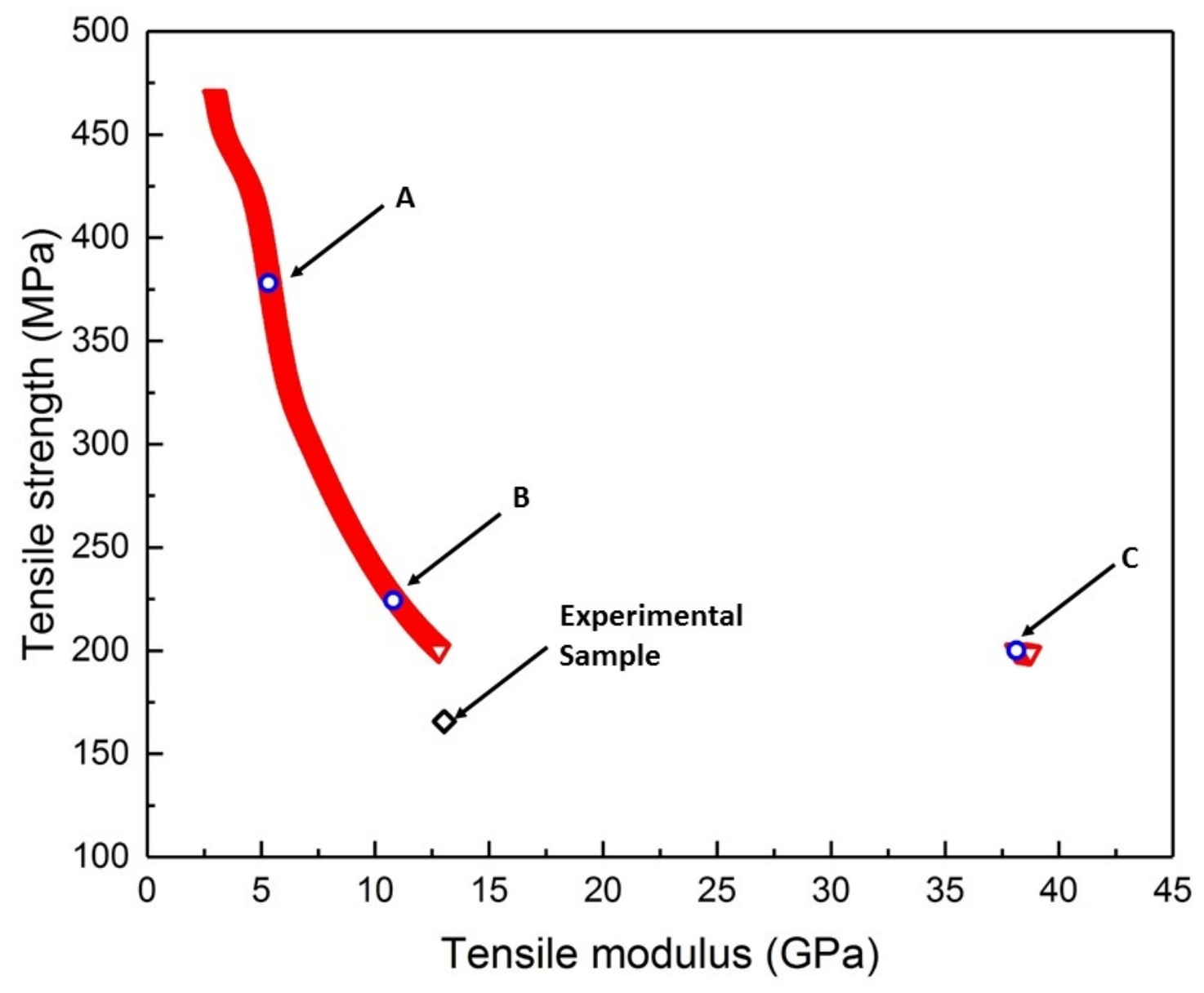

(a) 

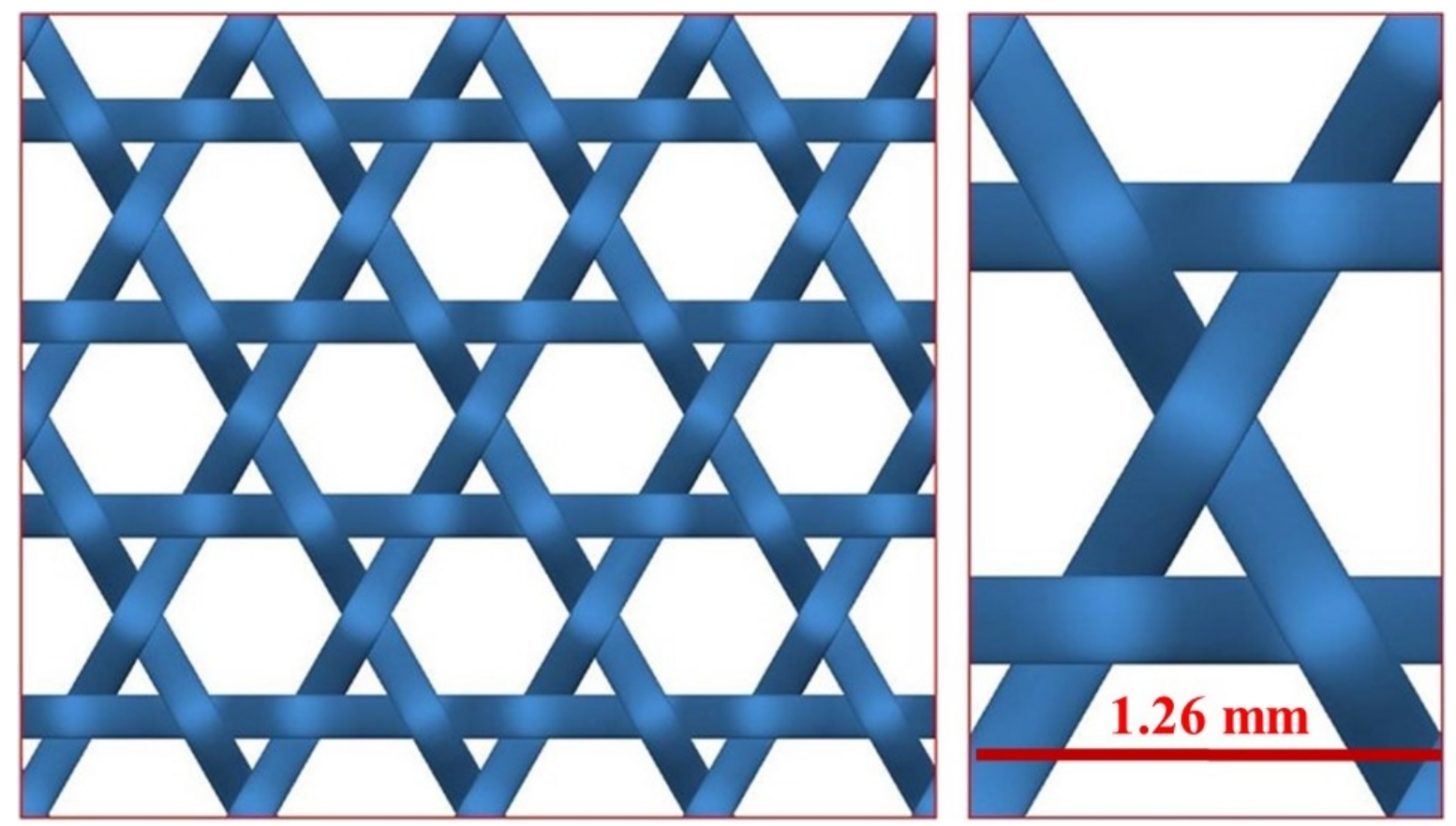

(b)
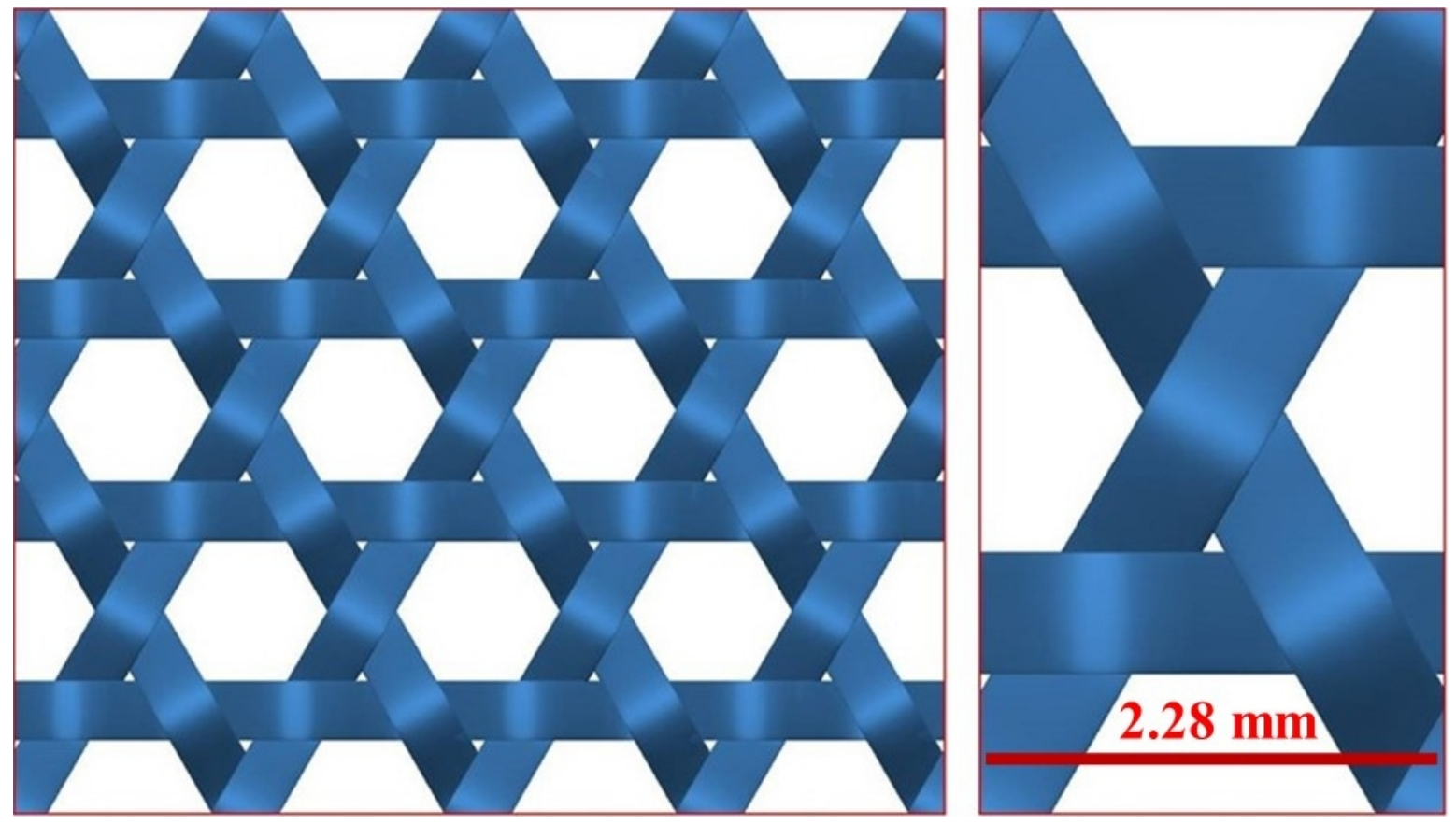

(c) 

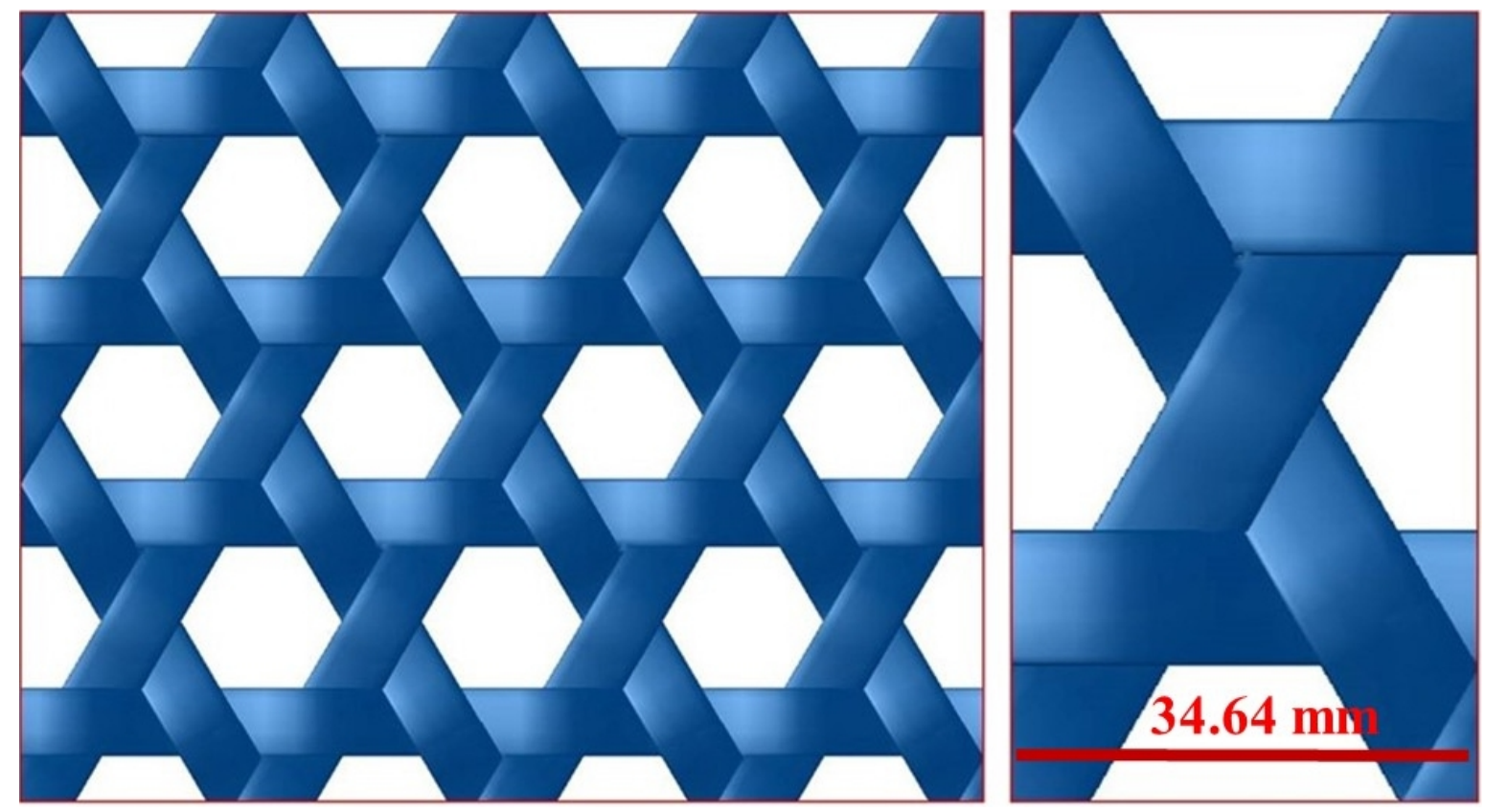

(d)

Fig. 6 Optimal designs of TWF composites: (a) sample points in Pareto front (b) Point A: $\mathrm{L}=0.63, \mathrm{w}=0.35, \mathrm{~h}=0.44$; (c) Point $\mathrm{B}: \mathrm{L}=1.14, \mathrm{w}=0.65, \mathrm{~h}=0.96$; (d) Point $\mathrm{C}: \mathrm{L}=17.32, \mathrm{w}=9.98$, $\mathrm{h}=0.94$

For the randomly selected points in Figure 6a, the left of Figures 6b, 6c and 6d, illustrate the weave pattern and the right shows the unit cell. The three sub-figures are at different magnifications, with a range of unit cell widths from $1.26 \mathrm{~mm}$ in $6 \mathrm{~b}$ to $34.63 \mathrm{~mm}$ in $6 \mathrm{~d}$. In Figure 6a, all of the left side Pareto front solutions are potentially applicable for ultralight structures under tension, with high strength to stiffness ratios which are suitable for producing flexible structures. Points A and B are representative of the general size of the points in the left side front. Compared with a current T300/Hexel8552 weave pattern [9], $\mathrm{L}=1.559, \mathrm{w}=0.803, \mathrm{~h}=0.78$, points A and B both have smaller unit cells; where the area of a T300/Hexel8552 unit cell is 612 times point $\mathrm{A}$ and 1.9 point $\mathrm{B}$. The bottom point of the left hand front, $\mathrm{L}=1.23, \mathrm{w}=0.71$, $\mathrm{h}=1.12$, with the lowest strength to modulus ratio of the optimised designs is improved by $28.80 \%$ compared to the experimental construction with the same modulus. The left hand front solutions above this provide higher strength to stiffness ratios where the top point shows an improvement of $1191 \%$. Between these two designs there are 643 points in the best Pareto front, providing a range of designs exhibiting different secondary properties. The tensile strength and modulus are related to all three variables, but are dominated by more compact weave patterns than the current T300/Hexel8552. The right side of the Pareto front provides results with significantly wider yarns and long undulation lengths, an example of which is point $\mathrm{C}$ 
illustrated in Figure 6d. The right hand materials are similar to Xu et al. [18] who performed a bi-axial buckling analysis of TWF composite structures with similar geometric parameters, indicating the potential for these weave patterns to resist compression despite poor tensile performance. The sample optimal designs, points A and B in Figure 6 illustrate triangular gaps in the unit cell. The extreme top point shows similar-sized triangular gaps as point B, but point A illustrate significantly larger triangular voids. Examples from the literature, [9-12], and the bottom point of the left hand front are similar to point $\mathrm{C}$, with no triangular gaps between the yarns. The size of triangular voids is controlled by the relationship between the undulation length and yarn width. These hexagonal and triangular holes help to reduce the load by increasing the penetrability of impacting air [18].

\section{Discussion and limitations}

There is 'no free lunch', improving an algorithms performance on a category of problems inevitably degrades its performance on other problem types. This results in specialist algorithms for a given problem that may perform badly on problems not of this type, and general solvers, with a lower performance on these specific problems but generally good performance across all the problems. The composite structures literature generally does not consider the problem type when selecting Genetic Algorithms for optimisation, meaning that general or outdated solvers are used in many cases. The complexity of the composite structural optimisation problems is getting larger and it has been shown in Mutlu et al. [1] to lead to unresolved Pareto fronts, even on simple problems. To investigate optimal designs of triaxial weave fabric composites four leading Genetic Algorithms are compared with the problem posed using both a constrained and an unconstrained formulation. MOEA/D, which in previous benchmarking provides top performance on unconstrained problems, shows the worst results on both formulations, which for the unconstrained results is unexpected. MTS also performs poorly on both formulation types, despite its excellent performance in benchmarking on both constrained and unconstrained problems. This demonstrates that these engineering problems are dominated by different characteristics than whether they are formulated as constrained or unconstrained; making current computational benchmarking unfit for selecting the correct algorithm in these cases. The results indicate that more composite structural optimisation problems need to be investigated, to determine the dominant features. It is likely that the literature increasingly will need to look at specialist solvers, rarely referenced in the current composite structural literature, but that these benchmarking exercises will more easily allow the selection of the correct algorithm and increase the use of these algorithms. 
For the TWF composite MLSGA-NSGAII and NSGA-II are the best performers, with MLSGA-NSGAII showing the greatest accuracy. NSGA-II cannot regularly find the optimal Pareto front on the left side but when successful it achieves the highest density of points. The NSGA-II mechanism is based on non-domination ranking through the whole population to find the Pareto front. Once part of the disconnected Pareto front is found, all of the solutions are pushed to this front and the possibility of missing the other parts of the front is increased. The crowding distance is used to maintain the diversity of solutions in NSGA-II and this indicates that the crowding distance is too weak to maintain diversity when the disconnected Pareto front has a large gap between two fronts. Based on these findings it is proposed that for NSGA-II the initial population determines the likelihood of success on disconnected objective spaces like this problem. MLSGA-NSGAII utilises its own collective evolutionary mechanism, incorporating separate crowding distances to keep the diversity of solutions. In MLSGANSGAII the population is divided into eight collectives meaning that different collectives find different parts of the entire front, though this leads to a reduction in the number of points found. The eight collectives are distributed throughout the search space giving it robust performance in finding the disconnected Pareto front. In this case it is proposed that the disconnected nature of the Pareto front favours MLSGA-NSGAII in both constrained and unconstrained formulations. This indicates that the disconnected nature of the front is the dominant characteristic of the search space but which isn't generally considered a dominant characteristic in the literature.

In addition to the algorithm selection, many composite structures papers obtain results from a single run. However, due to the stochastic nature of the solvers, it is demonstrated that it is necessary to run several cycles to ensure the entire Pareto front has been found depending on the nature of the objective space. Many algorithms converge on a solution early in the generation cycle. Using the computational time for a higher numbers of simulations, rather than fewer, longer runs, is likely to result in better solutions and resolved Pareto fronts. In this study increasing the population size also results in a higher possibility for finding the whole Pareto front, even with the same number of function calls. This goes against the prevalence of the micro-GAs, small population sizes with a large number of generations, often found in engineering to reduce the computational time.

The focus of the optimisation is tensile properties, but due to the number and diversity of points, 
designers should be able to find a suitable weave pattern matching their required secondary characteristics, such as low density or shear stiffness. To benchmark the different algorithms and to provide an ideal Pareto front, the size of the search space is artificially enlarged with gaps of $10^{-10}$ millimetres. However, to solve the problem practically the variable interval could be reduced to $10^{-2}$ millimetres. This will result in achieving the optimal results through fewer total function calls but the results here provide a more complete Pareto front for interest. A many-objective optimisation will provide more interest for designers than the bi-objective optimisation but is a significantly more complex problem to solve. Insight from the current optimisation will allow a method to be developed to include compression, shear and density properties into the design of triaxial weave fabric composites but will provide a refinement to the current selection due to the importance of the properties selected.

\section{Conclusions}

Triaxial weave fabrics (TWF) are increasingly used in novel ultralight applications and there is a requirement to improve material properties. The material properties are dependent on the weave pattern, so optimising the designs can lead to improved TWFs. In this paper Genetic Algorithms are used to find optimal weave patterns of TWF composites in tension. Since the dominant characteristics of the problem are not understood, four leading Genetic Algorithms specific to different problem types are compared to find the Pareto front for the TWF designs. Computational science literature defines constrained and unconstrained as the dominant features of an optimisation problem; with a recent benchmarking showing that MTS provides top performance on both problem types and MOEA/D on unconstrained problems. Poor performance from both of these algorithms demonstrates that this problem is dominated by different characteristics and that the selection of the correct algorithm is critical to find the optimal solutions. This indicates that it will be impossible to select the correct Genetic Algorithm for composite optimisation problems from the current literature. Therefore, there is a requirement for characterisation and benchmarking of composite optimisation problems to allow selection of algorithms capable of finding solutions to the more complex problems. The benchmarking demonstrates that for this problem MLSGA-NSGAII provides the best performance, providing a weave pattern which is seemingly dominated by the disconnected nature of the Pareto front. This process results in a left hand front which is suitable for producing flexible structures with 643 optimal designs. Of the proposed weave patterns one matching the modulus of a current experimental sample [9] gives an increase in the strength to 
stiffness ratio of $28.80 \%$. However, the lowest modulus solution achieves a greater tensile strength to stiffness ratio improvement of $1191 \%$ compared to the experimental sample.

\section{Acknowledgements}

This project was supported by the Lloyds Register Foundation, Chinese Scholarship Council and National Natural Science Foundation of China (Grant No. 51405006 and 51375033).

\section{References}

[1] Multi U, Grudniewski PA, Sobey AJ, Blake JIR. Selecting an optimisation methodology in the context of structural design for leisure boats, Design \& Construction of Super \& Mega Yachts, Genoa, Italy, May 2017.

[2] Zhang Q, Suganthan PN. Final report on CEC'09 MOEA competition. In Congress on evolutionary computation (CEC 2009), 2009.

[3] Bakar IA, Kramer O, Bordas S, Rabczuk T. Optimization of elastic properties and weaving patterns of woven composites. Composite Structures, 2013; 100:575-91.

[4] Axinte A, Taranu N, Bejan L, Hudisteanu I. Optimisation of Fabric Reinforced Polymer Composites Using a Variant of Genetic Algorithm. Applied Composite Materials. 2017; 1-13. [5] Fu X, Ricci S, Bisagni C. Minimum-weight design for three dimensional woven composite stiffened panels using neural networks and genetic algorithms. Composite structures, 2015; $134: 708-15$.

[6] Fu X, Ricci S, Bisagni C. Multi-scale analysis and optimisation of three-dimensional woven composite structures combining response surface method and genetic algorithms. CEAS Aeronautical Journal. 2017; 8(1):129-41.

[7] Nik MA, Fayazbakhsh K, Pasini D, Lessard L. Surrogate-based multi-objective optimization of a composite laminate with curvilinear fibres. Composite Structures. 2012; 94(8):2306-13.

[8] Bai JB, Xiong JJ, Shenoi RA, Zhu YT. Analytical Solutions for Predicting Tensile and Inplane Shear Strengths of Triaxial Weave Fabric Composites. International Journal of Solids and Structures, 2017; 120: 199-212.

[9] Kueh ABH, Pellegrino S. Triaxial weave fabric composites. Department of Engineering, University of Cambridge, June 30, 2007. 
[10] Aoki T, Yoshida K. Mechanical and thermal behaviours of triaxially-woven carbon/epoxy fabric composite. 47th AIAA/ASME/ASCE/AHS/ASC Structures, Structural Dynamics and Materials Conference, Rhode Island: Newport, May 1-4, 2006.

[11] Aoki T, Yoshida K, Watanabe A. Feasibility study of triaxially-woven fabric composite for deployable structures. 48th AIAA/ASME/ASCE/AHS/ASC Structures, Structural Dynamics and Materials Conference, Hawaii: Honolulu, April 23-26, 2007.

[12] Zhao Q, Hoa SV, Ouellette P. Progressive failure of triaxial woven fabric (TWF) composites with open holes. Composite Structures, 2004; 65(3-4): 419-431.

[13] Nguyen TT, Yao X. Benchmarking and solving dynamic constrained problems. In Evolutionary Computation, 2009. CEC'09. IEEE Congress on 2009 (pp. 690-697). IEEE.

[14] Deb K, Pratap A, Agarwal S, Meyarivan TA. A fast and elitist multi-objective genetic algorithm: NSGA-II. IEEE transactions on evolutionary computation, 2002; 6(2):182-97.

[15] Zhang Q, Li H. MOEA/D: A multi-objective evolutionary algorithm based on decomposition. IEEE Transactions on evolutionary computation, 2007; 11(6):712-31.

[16] Grudniewski PA, Sobey AJ. Multi-Level Selection Genetic Algorithm applied to CEC'09 test instances. In Evolutionary Computation (CEC), 2017 IEEE Congress on 2017 (pp. 16131620). IEEE.

[17] Tseng LY, Chen C. Multiple trajectory search for multi-objective optimization. In Evolutionary Computation, 2007. CEC 2007. IEEE Congress on 2007 (pp. 3609-3616). IEEE. [18] Xu D, Ganesan R, Hoa SV. Buckling analysis of tri-axial woven fabric composite structures subjected to bi-axial loading. Composite structures. 2007; 78(1):140-52. 


\section{APPENDIX A Samples of Optimal Designs}

\begin{tabular}{|c|c|c|c|c|c|}
\hline & $\begin{array}{c}\text { Tensile } \\
\text { Modulus } \\
(\mathrm{GPa})\end{array}$ & $\begin{array}{c}\text { Tensile } \\
\text { Strength } \\
(\mathrm{MPa})\end{array}$ & $\mathrm{L}(\mathrm{mm})$ & $\mathrm{w}(\mathrm{mm})$ & $\mathrm{h}(\mathrm{mm})$ \\
\hline 1 & 4.36 & 423 & 0.59 & 0.34 & 0.66 \\
\hline 2 & 4.63 & 417 & 0.60 & 0.35 & 0.54 \\
\hline 3 & 5.20 & 388 & 0.65 & 0.37 & 0.41 \\
\hline 4 & 6.02 & 332 & 0.75 & 0.43 & 0.47 \\
\hline 5 & 6.60 & 311 & 0.85 & 0.49 & 0.78 \\
\hline 6 & 7.05 & 298 & 0.94 & 0.54 & 1.14 \\
\hline 7 & 7.73 & 283 & 0.96 & 0.55 & 0.84 \\
\hline 8 & 8.12 & 274 & 1.00 & 0.58 & 1.04 \\
\hline 9 & 8.91 & 252 & 1.08 & 0.62 & 1.58 \\
\hline 10 & 9.52 & 245 & 1.08 & 0.62 & 1.06 \\
\hline 11 & 9.77 & 240 & 1.09 & 0.63 & 1.04 \\
\hline 12 & 10.0 & 236 & 1.10 & 0.64 & 0.98 \\
\hline 13 & 10.4 & 230 & 1.13 & 0.65 & 1.22 \\
\hline 14 & 10.8 & 222 & 1.14 & 0.65 & 0.94 \\
\hline 15 & 11.1 & 219 & 1.16 & 0.67 & 1.25 \\
\hline 16 & 11.6 & 213 & 1.18 & 0.68 & 0.98 \\
\hline 17 & 11.8 & 210 & 1.19 & 0.69 & 1.13 \\
\hline 18 & 12.1 & 207 & 1.20 & 0.70 & 0.94 \\
\hline 19 & 38.4 & 199 & 17.32 & 10.00 & 1.33 \\
\hline
\end{tabular}

